ppi $201502 Z U 4645$

Esta publicación cientifica en formato digital es continuidad de la revista impresa ISSN-Versión Impresa 0798-1406 / ISSN-Versión on line 2542-3185Depósito legal pp

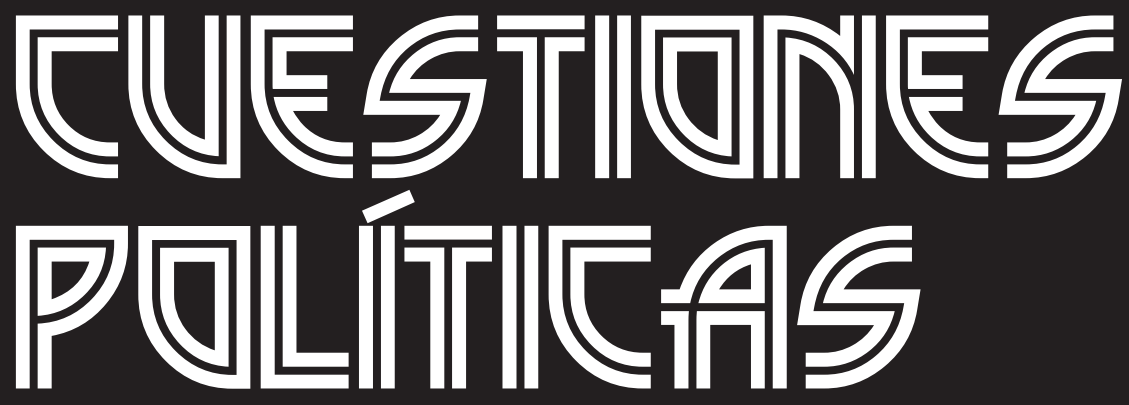

Instituto de Estudios Políticos y Derecho Público "Dr. Humberto J. La Roche' de la Facultad de Ciencias Jurídicas y Políticas de la Universidad del Zulia Maracaibo, Venezuela
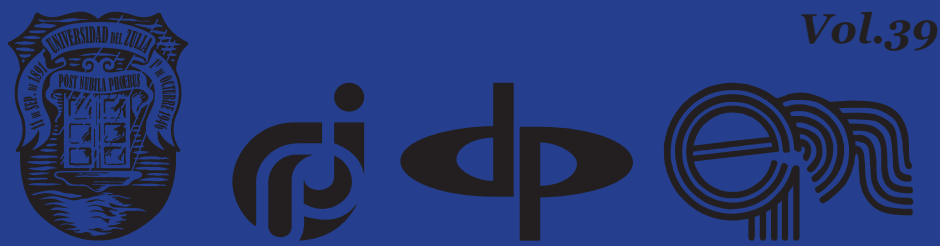


\title{
Neurocracia: la Democracia del Tercer Milenio
}

\author{
DOI: https://doi.org/10.46398/cuestpol.3968.58
}

\author{
Juan Guillermo Estay Sepúlveda * \\ Mario Lagomarsino Montoya ** \\ Juan Mansilla Sepúlveda *** \\ Rosalba Mancina-Chávez *** \\ Alex Véliz Burgos \\ Alessandro Monteverde Sánchez ${ }^{* * * * *}$
}

\section{Resumen}

La democracia es una quimera para muchos que sienten que ella jamás tocará sus puertas. Pero esa democracia ya es parte de un pasado al momento de ver como la ciencia avanza y el mundo empieza una brecha entre quienes tienen y quienes no tienen en todo el sentido de pensar y obrar del humano. En estos nuevos tiempos del milenarismo cibernético alimentado por las redes sociales, por un lado, y los laboratorios por otra, la nueva guerra por quien domina el pensamiento se librará a nivel de bits e Inteligencia Artificial. He aquí donde la neurocracia comienza su caminata como -quizásla nueva forma de vivir y convivir. El objetivo de este ensayo es dar a conocer como esta nueva forma de pensar, sentir y obrar de convivencia humana está ingresando en nuestro quehacer diario. Los resultados obtenidos apuntan a reafirmar que los mass media y la inteligencia artificial han llegado para quedarse en un escenario planetario cada día más distópico.

Palabras Claves:neurocracia; democracia; cybors; ética; análisis prospectivo.

* Universidad Católica de Temuco, Chile; Universidad de Salamanca, España; Universidad Adventista de Chile, Chile. ORCID ID: https://orcid.org/oooo-0oo1-7348-5529. Email: jges@usal.es

** Universidad Adventista de Chile, Chile; Fondazione Giovannino Pinna, Italia. ORCID ID: https:// orcid.org/oooo-0oo1-9204-4745. Email: mario.azzurro@gmail.com

*** Universidad Católica de Temuco, Chile; Universidad Pedagógica y Tecnológica de Colombia, Colombia. ORCID ID: https://orcid.org/oooo-ooo1-8175-7475. Email: jmansilla@uct.cl

****Universidad de Sevilla, España. ORCID ID: https://orcid.org/oooo-ooo2-4218-2338. Email: rmancinas@gmail.com

**** Universidad de Los Lagos, Chile; Fondazione Giovannino Pinna, Italia. ORCID ID: https://orcid. org/oooo-0003-1371-9041. Email: alex.veliz@ulagos.cl

***** Universidad de Playa Ancha de Ciencias de la Educación, Chile. ORCID ID: https://orcid.org/oooooo03-0172-2921. Email: amontev@gmail.com 


\title{
Neurocracy: Democracy of the Third Millennium
}

\begin{abstract}
Democracy is a chimera for many who feel that she will never knock on her doors. But that democracy is already part of a past when it comes to seeing science move forward and the world begins a gap between those who have and those who do not have in every sense of thinking and acting of the human. In these new times of social media-fed cyber millennialism on the one hand and laboratories on the other hand, the new war for those who master thought will be fought at the bit level and Artificial Intelligence. This is where neurocracy begins its journey as -perhaps- the new way of living and living together. The objective of this essay is to make known how this new way of thinking, feeling, and acting of human coexistence is entering into our daily work. The results obtained when thinking about the work, is of having shown that the middle maas and AI have arrived to stay in an increasingly dystopian planetary scenario.
\end{abstract}

Keyword: neurocracy; democracy; cibors; ethics; prospective analysis.

\section{Introducción}

1984 es una obra reveladora en cuanto a la creación por parte del Estado de una Policía del Pensamiento. En un instante de la historia de la segunda mitad del siglo XX aquel concurso de telerrealidad denominado el Gran Hermano se hizo realidad y las tele-pantallas nos inundaron a través de los diferentes dispositivos tecnológicos, con lo cual ingresamos a la medianoche del tiempo de más penuria del mundo: la noche de la era técnica (Heidegger, 1950). La inteligencia artificial es capaz de "leer" nuestro "pensamiento" a través de las tendencias y huellas que vamos dejando en los aparatos cibernéticos cuando navegamos7. Este gran Océano es el único que no tiene fosas y que su superficie se conoce y manipula permanentemente, incluso en la denominada "internet profunda" (Deep Web), sea en sus distintas versiones, a saber, Freenet, The Onion Router -Tor- y I2P, solamente por nombrar las más importantes y que permiten mantenerse en un anonimato de espejismos, ya que detrás de ellos, siempre hay alguien que sabe lo que hacen y también saben cómo manipular a los supuestos navegadores anónimos. El secretismo se supone que es la clave de la web profunda, "the most important institution of the Deep Web is anonymity. Each buyer and seller is known by a unique username; their true identity is secret"

7 Tal vez la única gran diferencia con la distopía de Orwell es que en aquella se trata de un régimen totalitario que espía y controla la vida en contra de la voluntad de las personas. Lo que sucede ahora es un control voluntario o relativamente voluntario sobre el cual los "ciudadanos son conscientes". En este sentido es importante revisar la obra de Armand Mattelart y André Vitalis. 2015. De Orwell al Cibercontrol. Editorial Gedisa. Barcelona, España. 
Juan Guillermo Estay Sepúlveda, Mario Lagomarsino Montoya, Juan Mansilla Sepúlveda, Rosalba Mancina-Chavez, Alex Véliz Burgos y Alessandro Monteverde Sánchez

(Hardy and Norgaard, 2016: 516). Se: "Estima que el tamaño de la internet profunda es 500 veces más grande que la web superficial; esto significa que los buscadores tradicionales no rastrean el 99\% del contenido existente en internet” (Sánchez, 2015: 105). En síntesis,

The darknet (aka "dark address," "lost net," "dark address space," "greynet," "sparse darknets," etc.) consists of a computer network characterized by an anonymous and restricted access by third parties, mainly used for illegal and/ or criminal activities. The darknet is a subsection of the deep web, that is, the World Wide Web content hidden and not accessible by means of standard search engines, consisting in password protected or dynamic websites as well as encrypted networks (Orsolini et al., 2017: 3).

Las Bestias de Inglaterra (Rebelión en la Granja) traspasaron las fronteras de la isla europea, para transformarse en un Leviatán que devora todo a su paso a través de las tecnologías desarrolladas y profundizadas en el siglo XXI. Ya no es la granja de Jones, sino, las grandes corporaciones privadas (para el mundo occidental) y estatales (para el mundo oriental) las que nos dicen en elocuentes carteles que algunos seres humanos -los mínimos- son más iguales que otros seres humanos -los mayoritarios-. Sin embargo, esta tentación verticalista del poder atrae a gobernantes de Occidente que anhelan el control de la población, una vocación de conciencia jerarquizada complejo de autoridad sagrada, que hunde sus raíces en la transición desde el Paleolítico al Neolítico (Berman, 2006).

Este deseo choca con la libertad individual de creación y las libertades que los seres humanos protestando en calles y -paradójicamente- en las redes sociales. Se actualiza la tensión entre igualdades y libertades tal como lo pensó y escribió en su hora Alexis de Tocqueville (De Tocqueville, 1856/2015). Leviatán para unos y para otros, si aplicamos una visión individualista, pesimista y en algunas partes realista. El poder en manos de unos pocos pensando individualmente y la libertad que se encuentra en cada ser humano como ADN imposible de fracturar. Las principales creaciones de Medios de Comunicación Masivo (MCM) han nacido de individuos y no de Estados. Orwell es categórico para entregar una voz de alarma a la población y su vida y privacidad de cada una de las personas que habitan este planeta. Estamos a un paso de la manipulación humana.

He aquí donde la Democracia hace su entrada, ya no como la ateniense de Pericles o la de la época de las Grandes Revoluciones de la Edad Moderna, ni de la participación primaveral de las calles que nacen en el norte de África y Oriente Medio para irradiar al resto del orbe. Quizás ni siquiera como aquella de la "Voz Populi, Vox Dei" que hizo caer monarquías y gobiernos actuales. Se debe explorar la idea de una nueva democracia para dar respuesta a las nuevas tecnologías, se debe dejar abierto el camino a la neurocracia y establecer sus deberes y derechos como Tablas de la Ley que deben no ser violadas. Incluso ir más allá. 
No en una Declaración de Derechos Humanos que no es vinculante a los Estados como la actual de la Organización de Naciones Unidas, sino que, una normativa de la humanidad vinculante a todo Estado y territorio que componen nuestro planeta. Y con ello, como se ha planteado en anteriores investigaciones y ensayos, la democracia pese a todos sus errores es el único camino que nos queda en estos tiempos de fundamentalismo de todo orden (Estay Sepúlveda y Lagomarsino, 2020)

\section{Neurocracia: la Democracia del Tercer Milenio}

Los neuroderechos se encuentran en discusión en la actualidad (Borbón et al., 2020). La neurocracia se avizora como la nueva forma de velar y proteger nuestros derechos, a la vez que, ante ciertas circunstancias, se solicite en un futuro no lejano y bajo norma judicial o de razón de Estado, des-velar y des-cubrir que sucede en la mente de cada una de las personas. En consecuencia:

For over 2000 years, western thought has debated whether free will and responsibility are possible if determinism, universal causation or the like is true. The deterministic explanations have shifted with changes in theological and scientific understanding and fashion. God's foreknowledge, social structure, unconscious psychodynamics, behavioral psychology, and genetics have all been seen as the basis for determinist understanding. Neuroscience is simply the newest alleged source of determinism on the block. Despite such changes, the alleged incompatibility between determinism and responsibility is an ancient issue. In this debate, free will is usually understood as the ability of people to act uncaused by anything other than themselves. If people do not have this ability, it is claimed, responsibility and other worthy goods such as autonomy may be unjustified. This thought is what disturbs people about scientific understanding of human behavior, which relentlessly exposes the numerous causal variables that seem to toss us about like light ships in a raging sea storm. Neuroscience, it seems, will finally support this challenge because it exposes that the brain, the final pathway to action, is nothing but a mechanism (Morse, 2016: 16).

Adelantarse a los acontecimientos no es ciencia ficción, como quedó demostrado en la película Minority Report del año 2002. Las neurotecnologías, por ejemplo, han demostrado experimentos de estimulación a través de las interfaces cerebro-máquina, las cuales: "Pueden cambiar aspectos de la personalidad o del "yo" e incluso, "como tienen como objetivo áreas específicas del cerebro, durante su uso pueden alterar los estados de ánimo, los deseos, la conducta e incluso los valores y la identidad personal" (Monasterio et al, 2019: 33).

Incluso, investigadores del Instituto de investigaciones de Telecomunicaciones Avanzadas en Japón se han preguntado si una prótesis controlada por el pensamiento que trabaje junto con los brazos biológicos de una persona puede dar a esa persona habilidades de multi-tarea superiores 


\section{Juan Guillermo Estay Sepúlveda, Mario Lagomarsino Montoya, Juan Mansilla Sepúlveda, Rosalba Mancina-Chavez, Alex Véliz Burgos y Alessandro Monteverde Sánchez

a las de una persona media (Monasterio et al, 2019: 33). Recientemente, aplicaciones como spotify, obtienen patente para poder "espiar", "basic information such as gender or age", donde:

At one point, the patent suggests that obtaining "intonation, stress, rhythm and the likes of units of speech" could be combined with "acoustic information within a hidden Markov model architecture" so that Spotify's app could categorize a user's mood as "happy, angry, sad or neutral (musicbusinessworldwide.com, 2021, s/p.)

$\mathrm{Y}$, recientemente investigadores se encuentran analizando la funcionalidad del cerebro humano a través de experimentos con minicerebroides con la intención de recrear el cerebro de los neanderthales y con ello, aproximarse a saber o inferir como era su forma de pensar y obrar y, por ende, su forma de vida. De ahí el salto a comprender nuestro cerebro de sapiens sapiens es algo no alejado en el tiempo (Trujillo et al., 2021).

Sin dudas, los beneficios para las personas y la humanidad son de una trascendencia única. Pero al mismo tiempo, la cantidad de millones de dólares que se mueven son estratosféricos

Among less invasive technologies, some devices remain on the surface of the human body - these are usually called wearables. Wearable technologies are a fast expanding area, with a $\$ 15.74$ billion market in 2015, estimated to grow to $\$ 51.60$ billion by 2022. Electronic skin patches alone, which are widely adopted in medical wearables for cardiovascular monitoring, diabetes management, temperature, sweat and motion sensing and othertypes of biomarker monitoring, achieved more than $\$ 7.5$ billion in revenue in 2018. Non-medical wearables are a dynamic field, with products and adoptions ranging from personal fitness trackers and smartwatches to enterprise applications (Liu y Merritt, 2020: 8)

En la actualidad, movimientos como "Quantifified self" utilizan la tecnología para adentrarse en los datos de las personas, aunque sean los mínimos, bajo la justificación del bienestar (Monasterio et al, 2019: 35) y bajo la lógica ética de usar esos datos para el desarrollo saludable y de entretención humana. La cantidad de información electrónica en la actualidad es impensable

Sobre el tema, académicos de los macrodatos (Big Data en inglés), en particular Martin Hilbert quien fuera asesor de la Comisión Económica para América Latina y el Caribe (CEPAL), planteó que los datos que se generan en internet por año son superiores a los los 9 zettabytes, lo que él explica serian unas 9000 pilas de libros desde la tierra hasta el sol.

También se podría entender que la generación de esos datos anuales equivaldría aproximadamente, a los de una película HD con más de 350 millones de años de duración. Ante tal panorama, los algoritmos son herramientas potentes e indispensables para el procesamiento de toda esa información y para la automatización de servicios que se pueden ofrecer al ciudadano, el problema está en la orientación y en el necesario uso ético que se debe hacer de las mismas (Gómez, 2020: 2). 
Es aquí donde hace ingreso la corriente DIYbio, la cual bajo la premisa de la libertad y la individualidad, puede llegar a causar mayores daños que beneficios, y quienes se encuentran sumergidos y desarrollando el nuevo milenio son: "diverse sets of individuals such as scientists, designers, software developers, hobbyists, and enthusiasts, that work on a wide range of projects such as citizen science initiatives, amateur science, product development (incubators), artistic work, and educational workshops and courses" (Sánchez, 2014: 23).

Si bien es cierto, no todos los que pertenecen a este grupo acepta el término biohackers -por su connotación negativa (delincuente cibernético)-, otros si lo aceptan. Quienes se han adentrado al estudio de ellos y sus acciones y un buen número de quienes pertenecen al movimiento, llegan a la conclusión de que "biohackers want to open access, or in other words: democratize biology as knowledge, as a science, and as a technology" (Sánchez 2014: 50). Es de esperar que la ética realmente sea el motor principal y fundamental de quienes se encuentran en la corriente DIYbio y no caer en las "garras" de los inescrupulosos de siempre provenientes del mundo de las finanzas, la política y las armas letales.

A new model of $\mathrm{P} 2 \mathrm{P}$ science is already making its way in institutional science with initiatives such as open science, citizen science, and open innovation, where they establish close cooperation and funding mechanisms with DIYbio. Whether their support comes from legitimately recognizing DIYbio as an low-cost and inclusive solution to education and innovation, or as an exploitation of cheap cognitive capital remains unclear and is yet to be seen. What is certain is that DIYbio will have to develop strategies to take advantage of new opportunities created, as they navigate between the old model and the new model(CBPP), while preserving the ethos of the movement. Such case would be how the movement will manage government and corporate funding whose interests might encroach on their freedom of inquiry by dictating projects or suppress their commitment to openness and sharing by demanding IP protection for commercial exploitation. (Sánchez, 2014: 51).

El crecimiento de las tecnologías es exponencial al pensamiento. La idea de lo que pensamos está años luz de las fronteras de la técnica, parece cada día más una quimera fácil de desmentir. El big data ha dejado atrás a los astrólogos y adivinos y libros como Las Centurias de Nostradamus son juguetes de niño recién nacidos para los ingenieros cibernéticos. En estas dos décadas que van del nuevo milenio, se observó a través del mundo e internet -que ironía- como las personas tomaban decisiones a través de likes y me gusta (Norte, 2020). Quienes tienen acceso al manejo de los datos, tendrán acceso al manejo de las vidas y de ahí a una cybercracia, datacracia y/o neurocracia se encuentra a la vuelta de la esquina. El decidir ya no se encuentra a nuestro albedrío (Jurno y D’andrea, 2017).

La neurocracia, como la democracia, debe cuidarse del lenguaje para no convertirse en el Nuevo Oficio de una Santa Inquisición de las Masas y 
Juan Guillermo Estay Sepúlveda, Mario Lagomarsino Montoya, Juan Mansilla Sepúlveda, Rosalba Mancina-Châvez, Alex Véliz Burgos y Alessandro Monteverde Sánchez

los Gobernantes. En ello, el lenguaje produce realidad. Sea este lenguaje verdadero o una noticia falsa (Fake News). Al final, produce una realidad que lo tomará un sector de la población en mano de los mass media y con sus like (en Instagram) o los "me gusta" (en Facebook) los legitimarán, sin ni siquiera profundizar en la noticia o en verificar lo que se está comentando, o lo peor, sin leer lo que dan como visto bueno o haciendo una pira para quemar al que todos queman sin saber que están quemando y por qué lo están consumiendo en las llamas.

Total, que importa. Si hubo un error, serán los mismos que aprietan los botones del me gusta y el like, los que criticarán a quien dio a conocer lo falso. Asunto concluido. Pero, el quemado ya se consumió, tal cual Damiens en Vigilar y Castigar (Foucault, 2009).

Desde finales del siglo XX conocemos la existencia de otro tipo de campañas de desinformación a gran escala relacionadas con el cambio climático, las vacunas, los alimentos, la nutrición, el origen de la vida, la salud, las armas en manos de los ciudadanos, los medicamentos genéricos, la curación u origen de enfermedades, la energía nuclear o el impacto de la inmigración. Pero sin duda, el momento más álgido del fenómeno de las fake news se vivió a partir de dos acontecimientos que sucedieron en 2016: el proceso del Brexit en Reino Unido y las elecciones norteamericanas que ganó Donald Trump. Una de las numerosas investigaciones realizadas a partir de estas últimas, confirmaba que durante la campaña electoral se generaron un total de 115 noticias falsas favorables al actual presidente de Estados Unidos que se compartieron en Facebook un total de 30 millones de veces, frente a las 41 fake news en beneficio de Hillary Clinton compartidas en 7,6 millones de ocasiones (Parra y Oliveira, 2018: 55).

Enormemente llama la atención que, en la elección del año 2016 en Estados Unidos, "las noticias falsas alcanzaron casi los nueve millones de visitas mientras que las noticias generales verdaderas un millón y medio menos" (Salas, 2019: 422). Un estudio de IPSOS del año 2018 demostró que a nivel mundial los habitantes de los países que más han creído en noticias falsas son los brasileños (67\%), sauditas (58), surcoreanos (58\%), peruanos (585 y españoles (58\%), dentro del top cinco (Muñoz-Machado, 2020: 124).

La democracia bajo esta nueva lógica de ver el mundo por sus habitantes se ve amenazada. Más aún, cuando los que están elegidos para cuidarla, cometen errores conscientes. En este mundo del tercer milenio, el homo economicus con su semejante homo consumens han derribado las barreras de que dos tipos de homo no puedan convivir. Incluso, esta nueva forma de ver y obrar en el mundo está consumiendo las relaciones más íntimas del ser humano: la relación de pareja. En ese sentido, Bauman tiene razón al llamar a esta nueva forma de convivencia: amor líquido (Bauman, 2005).

Sin embargo, la democracia no ha perdido su clasismo de élite. No compartimos la idea de Liberos et al., (2013) de que las nuevas tecnologías vinieron a democratizar el mundo, en el sentido que democracia es igual a 
opiniones de masas y que cada individuo tiene voz y voto en este mundo. Si bien es cierto, el anonimato individual ha desaparecido y cada cual es un ser con opinión, el correr del tiempo ha demostrado que este "espejismo democrático" se encuentra titiritado por quienes ostentan verdaderamente el poder. Más aún, cuando la población se une a temas de discusión política en forma fragmentaria y sin conocer el contenido completo de una conversación o tema o como muy bien lo desarrolla Valera-Ordaz (2019), al argumentarlas que son espontaneas.

Los mass media nos indican que pensar, actuar y obrar. Con esto no planteamos que la democracia no sea el mejor entre los peores regímenes: es lo mejor que se ha inventado y planteado para el mundo occidental en los últimos veinticinco siglos desde su nacimiento en la vieja Atenas. Solamente cuestionamos que ese espíritu que la sustenta no ha llegado a todos. Sigue siendo demos y no laikos.

$\mathrm{Al}$ mismo tiempo, la neurocracia puede producir castas o clases. Los neuroenhancement-man ${ }^{8}$ al poseer el poder adquisitivo para desarrollar su sapiensa, sabrán como dominar al resto de la población o seguir dejándola en este nuevo milenarismo del tercer milenio, donde la literatura de dudosa reputación se abre paso entre las personas.

A un nivel más político, el neuroenhancement radicaliza y lleva casi a un nivel de ciencia ficción las antiguas discusiones sobre desigual distribución de los bienes, al producir desigualdad e incrementando la segregación cuando aquéllos que puedan adquirir smart drugs se encuentren mejor situados cognitivamente frente a quienes no las consumen, lo que lleva a una ventaja injusta de uno sobre otros, e incluso una "selección artificial». Este problema de justicia social posee también un dilema ético, en cuanto aquéllos que están en una posición ventajosa estarían cometiendo plagio o «dopaje intelectual» (Cornejo, 2019: s/p).

En el mismo sentido, si somos capaces de interferir en la mente humana ¿quién nos dice que no podamos hacerlo en un crimen y poder manipular las pruebas?, incluso la de los neuroabogados y la neurojurisprudencia. En este sentido,

Sí existe la libertad, pero no es como creíamos», en el sentido de que «nuestras decisiones pueden ser fruto de nuestra morfología cerebral y de las necesidades adaptativas del cerebro según las vivencias personales que van pasando por nuestra vida», pero «esos impulsos no son ni omnicomprensivos ni unívocos, en el sentido de que la respuesta a un estímulo tiene diversas posibilidades igualmente elegibles, y además es posible que nuestro cerebro carezca de respuesta ante algunas situaciones (Rodríguez, 2018: 197).

Recientemente, el 6 de enero de 2021 observamos como en un "nuevo país bananero", el clímax llegó con un ataque a la House of People (El

8 Nominación dada por los autores de este papers a las personas que quieren o querrían desarrollar sus capacidades cognitivas a través de la biociencia. 
Juan Guillermo Estay Sepúlveda, Mario Lagomarsino Montoya, Juan Mansilla Sepúlveda, Rosalba Mancina-Chăvez, Alex Véliz Burgos y Alessandro Monteverde Sánchez

Capitolio) lo que hizo que quienes prestaron sus plataformas para alimentar teorías conspirativas hicieran realidad el aforismo de Pierre Victurnien Vergniaud: "La revolución acaba devorando a sus hijos". Facebook, Twitter, Youtube, Instagram, Google, Whatsapp -solamente por nombrar algunos dispositivos de control-, decidieron cerrar las cuentas de quien en ese instante incitaba a sus seguidores a marchar: "We're going to walk down Pennsylvania Avenue... and we're going to the Capitol... we're going to try and give our Republicans ... the kind of pride and boldness that they need to take back our country" (limaohio.com, 2021: s/p).

Durante cuatro años, bajo el argumento de la libertad de expresión y teniendo bajo el brazo la Primera Enmienda, no hicieron nada y a pocos días del abandono de la Casa Blanca de Donald Trump -en un arranque delirante de civilidad-, decidieron en pos de la democracia comenzar a criticar los mensajes de odio. Mensajes de odio que no censuraron antes. En un par de minutos, irónicamente todos se convirtieron en seguidores de Karl Popper en defensa de la democracia y con ella, tomar decisiones no democráticas -la libertad de expresión censurada- para proteger la propia democracia.

$\mathrm{Y}$, eso lo hicieron porque la revolución comenzaba a comerse a sus hijos y Silicon Valley no se vería bonito con muchedumbres paseando por sus jardines. Los congresistas en ese momento ocultándose debajo de sus asientos o escapando del hemiciclo, los sentarían -más temprano que tardea rendir cuentas. Esta realidad estaba anunciada en los libros, películas, series y música de ciencia ficción y en la no ficción. Mundos diptópicos se estaban creando:

Zuckerberg, ao destacar que sua rede social tem como objetivo "projetar experiências"em seus usuários, elabora uma plataforma com a potência distópica contemporânea de controle político-cognitivo, podendo atuar como regime autorrepresentativo e de visibilidade pública de si mesmo, mesclando os tempos reais e virtuais, hibridizando em si o espaço-tempo e a esfera público-privada, blindando, por consequência, possibilidades disruptivas de questionamento, criação e transformação inerentes à cultura (Madureira, 2019: 135).

Situacionesquepuedansermanejadasalantojodelosneuroenhancementman. No estamos hablando -por el momento- de un futuro apocalíptico, pero la libertad del ser humano debe ser defendida contra viento y marea. La Inteligencia Artificial -IA- es bienvenida y necesaria para el desarrollo de la humanidad (Gallina, 2019; Barrat, 2013; Perucchietti, 2019). Lo cyborg es algo existente. La pregunta es si ésta IA estará para ayudar a los seres humanos en su trascendencia y sobrevivencia o se cansará de sus creadores. Pensar hoy en día, junto a Philip K. Dick si ¿̇sueñan los androides con ovejas eléctricas?, podríamos decir que estamos a un paso de violar las tres leyes de la robótica de Asimov. 
La dictadura de las plataformas digitales se encuentra en su auge. Las plataformas a través de sus millonarios recursos podrán decidir qué hacer y no hacer con nuestras vidas y poder luchar contra grandes multinacionales será como luchar contra el poder de la nueva plutocracia (Gillespie, 2018). Las nuevas tecnologías "podem ser cerceadoras e castradoras da capacidade de indivíduos e grupos de existir e agir" (Veloso y Lopes, 2020: 167). Quien no se encuentra conectado incluso puede sufrir el síndrome FoMO.

El acceso a las plataformas de redes sociales y al consumo libre, el nuevo perfil de consumidor, hiperconectado y activo en redes sociales han abonado el terreno para fenómenos enfrentados de libertad y sometimiento; la libertad para elegir qué información consumir, difundir noticias y opiniones, generar contenidos, vincularse a grupos afines y por otro lado el sometimiento a la datacracia, la infoxicación, la posverdad y las fakenews, determinados en gran medida por fenómenos psico-sociales vinculados al FoMO (fear of missing out) y al sesgo de confirmación. Los medios dan servicio a mediadores con capacidad de influir en las audiencias en un contexto en el que hoy en día cualquiera puede apropiarse y difundir la noticia. Los datos son el principal interés de la nueva economía, monopolizados por unas pocas plataformas que ofrecen servicios gratuitos con la contraprestación de adquirir nichos de poder alimentados por los datos y su extracción, análisis y comercialización en el denominado "capitalismo de plataformas"

Nos enfrentamos a un contexto en el que las plataformas que median en la comunicación entre personas, o Behavioral Modification Empires, están tratando de modificar su conducta. Lo mismo sucede cuando abrimos una aplicación para consumir su contenido, donde las más o menos sutiles recomendaciones algorítmicas se ponen al servicio de incrementar nuestra interacción y la permanencia en la plataforma (Hernández, 2020: 222).

Esta nueva forma de manipulación es en tiempo real (Castellanos, 2019) y puede realizarse simplemente a través de los algoritmos y una que otra compañía que esté disponible, como lo hizo Cambridge Analytica a través de macrodatos (Betzu et al., 2019) y que fue utilizada en campañas electorales de los últimos años y que dieron, por ejemplo, un posicionamiento de Trump en las redes sociales, apuntando directamente al ciudadano medio estadounidense y con una campaña logísticamente planificada (Ayala, 2017). En palabras de Castellanos, "La democrazia pare aver mutato il proprio DNA, e la fonte di questa mutazione è la rivoluzione digitale. La politica ormai si fa sugli smartphone, a colpi di post allarmistici, tweet mattutini e selfie con gli elettori" (Castellanos, 2020: 42).

Los algoritmos y su brazo armado de la publicidad y los medios de comunicación son herramientas esenciales para la propaganda en el mundo de las campañas electorales (Leal, 2021), entendiendo dicha propaganda computacional: "Como la concurrencia de redes sociales, agentes autónomos de la IA y el tratamiento del Big Data, cuyo objetivo es 
Juan Guillermo Estay Sepúlveda, Mario Lagomarsino Montoya, Juan Mansilla Sepúlveda, Rosalba Mancina-Châvez, Alex Véliz Burgos y Alessandro Monteverde Sánchez

906

Neurocracia: la Democracia del Tercer Milenio

la manipulación de la opinión pública” (Petit, 2018: 6).

Todo ello a través del microtargeting, que será una operación microscópica directa al individuo. Una cirugía perfecta y exacta al pensamiento de la persona, que se obtuvo gracias a sus navegaciones por la red. He aquí el efecto burbuja es ama y señora del pensar y obrar del individuo y, por ende, de los individuos, "cuando en Facebook un usuario efectúa una acción tan aparentemente inocente o neutral como la aprobación de un contenido, la "manita con el dedo pulgar hacia arriba, que indica que me gusta" esos datos se pueden recoger con gran facilidad y de forma automatizada para elaborar psicometrías" (González de la Garza, 2018: 281-282). Del mismo modo, podemos estar charlando con sockpuppet o un troll sin darnos cuenta (Gorwa y Guilbeault, 2018). La dictadura de las plataformas es una dictadura que incluso se puede adelantar a lo que pensamos a través de cruce de algoritmos.

Desse modo, as plataformas das redes e mídias sociais como o Twitter, $e$ as práticas que elas favorecem, também são componentes do dispositivo de vigilância - um conjunto que, além de textos, discursos, protocolos de rede, imagens, vídeos, tecnologias, empresas e leis, inclui agentes não humanos como os algoritmos dos buscadores que indexam as páginas na web, os sistemas de recomendação das mídias sociais, os cookies que acompanham e rastreiam a trilha de dados deixada pelos internautas e, também, robôs de conversação como Tay (Vale, 2016: 40-41).

Iniciativas comunitarias para acabar -no regularizar- con las noticias falsas en líneas y la manipulación que existen detrás de ellas, han sido tomadas, por ejemplo, por Unión Europea, donde se propone:

Mejorar la transparencia de las noticias online, promover la alfabetización mediática e informativa, desarrollar herramientas para empoderar a usuarios y periodistas, salvaguardar la diversidad y la sostenibilidad del ecosistema de los medios de comunicación europeos y promover la investigación continua sobre el impacto de la desinformación en Europa (Aba-Catoria, 2020: 136).

Para el caso de América Latina, al encontrarnos en un Feudalismo Digital (Ávila, 2018), el pasar de una colonización a otra ya es un acto que pareciera imposible de sacudir. En resumen, si bien Cambridge Analytica dejo de existir, "já existiam e ainda persistem indícios de novas empresas de análise comportamental eleitoral no mundo" (Fornasier y Beck, 2020: 189), que tendrán a personas o instituciones/organizaciones dispuestas a comprar esos mecanismos de manipulación electoral, donde:

La posibilidad de los operadores de Internet de procesar la información de sus usuarios y crear perfiles muy precisos de los mismos, además de predecir sus preferencias e incluso dirigirse a ellos con datos y publicidad individualizados, consiguen promover o desalentar determinadas conductas, entre las que se encuentra la decisión de voto (Arena, 2019: 350). 
E incluso, pasado el período electoral, influir "en el proceso de formación de la voluntad de los ciudadanos" (Arenas, 2019: 350), donde además se "pueden utilizar los avances tecnológicos de forma permanente en su función de formación de la opinión pública" (Arenas, 2019: 350). Debemos estar conscientes que, en la actualidad, podemos estar conversando un tema sin darnos cuenta de que, quien se encuentra al otro lado de la pantalla, no es otro ser humano, sino un bot que es capaz incluso de contestar en forma irracional:

This ability to carry on conversations at length is what makes the technology behind XiaoIce - and Tay-different from previous chat bots. Like earlier chat bots Eliza and Parry, XiaoIce and Tay use strategies of deflection and indignation when faced with difficult-to-answer questions. But unlike those bots, XiaoIce and Tay have intentionally built-in "human" conversational qualities such as unpredictability and irrationality. XiaoIce offers resistance to her conversation partner at several junctures, has clear opinions, and is often capricious (Neff y Nagy, 2016: 4920)

Tay es un caso muy especial de analizar. Desarrollado por Microsoft en 2016, tuvo que ser desactivado al poco tiempo debido a que "aprendió" a insultar y proferir frases discriminatorias contra los usuarios, las cuales las fue adaptando a "su cerebro" gracias a su interacción con los usuarios de Twitter. Lograr en la actualidad ir descubriendo bots se hace cada vez más difícil, a medida que las tecnologías se van desarrollando a una alta velocidad (Murthy et al., 2016). Los bots, en este sentido, han hecho una excelente tarea en los planos de la política y el comercio y las finanzas.

\section{Conclusiones}

Cuando George Orwell escribe su obra distópica 1984 criticaba el sistema totalitario en los países comunistas. Un Estado fuerte, omnipresente, capaz de controlar hasta las acciones más íntimas de los ciudadanos. Enfrente estaba el sistema capitalista, la economía de mercado que ofrecía como valor esencial la libertad de los individuos para llegar a donde quisieran llegar, libres del poder del estado, libertad de expresión, libertad de empresa, libertad, por encima de todo lo demás.

Con el avance de las tecnologías de la información y la irrupción de la Inteligencia Artificial como una realidad cotidiana, el futuro ha llegado y estamos viviendo esa sociedad distópica que fue tan novelada en el siglo XX, y que es capaz desestabilizar a Estados (Rosenbach y Mansted, 2018) y de estar en una frágil línea de traspaso para la violación de la privacidad (Boehme-Neßbler, 2016).

El ser humano en su libertad ha avanzado hacia un sistema totalitario que lo controla todo. Con una enorme ventaja, el poder es invisible, no hay enemigo a vencer, no existe un Estado malvado a quién derribar. La falta 
Juan Guillermo Estay Sepúlveda, Mario Lagomarsino Montoya, Juan Mansilla Sepúlveda, Rosalba Mancina-Chàvez, Alex Véliz Burgos y Alessandro Monteverde Sánchez

908

Neurocracia: la Democracia del Tercer Milenio

de un enemigo común y la continua propaganda a favor del individualismo exacerbado ha llevado a la atomización de la sociedad en esta nueva etapa de la neurocracia. Esta nueva realidad exige a los estudiosos de las humanidades y las ciencias sociales adoptar nuevas fórmulas para el análisis, la dicotomía simplista de buenos o malos ya no resuelve las interrogantes planteadas a lo largo de este trabajo, exige avanzar hacia un modelo de estudios que Reig (2020) denomina Enfoque Estructural Complejo (EEC), siguiendo los planteamientos de pensamiento complejo de Edgar Morin (1998).

En esta etapa de la evolución humana, los planteamientos básicos de las humanidades y las ciencias sociales tendrán que abrir su perspectiva a otras ciencias, las matemáticas, la física y la biología, fundamentalmente, para comprender el comportamiento de los individuos como seres que funcionan en masa y se manipulan a través de algoritmos programados con determinada finalidad. La neurocracia es la etapa más reciente de la sociedad de la información, como se decía en el siglo XX, la información es poder, pero no imaginábamos hasta qué punto llegaría este poder al tener el control de los datos.

\section{Referencias Bibliográficas}

ABA-CATORIA, Ana. 2020. "Los desórdenes informativos en un sistema de comunicación democrático” En: Revista de Derecho Público. No. 109, pp. 119-151.

ARENAS RAMIRO, Mónica. 2019. "Partidos políticos, opiniones políticas e internet: la lesión del derecho a la protección de datos personales" En: Teoría y Realidad Constitucional. No. 44, pp. 341-372.

ÁVILA, Ramon. 2018. “¿Soberanía digital o colonialismo digital? Nuevas tensiones alrededor de la privacidad, la seguridad y las políticas nacionales” En: SUR, Vol. 15, No. 27, pp. 15-28.

AYALA SÁNCHEZ, Alfonso. 2017. "Análisis de la campaña electoral de Trump desde el enfoque de la teoría democrática" En: Justicia Electoral. Vol. 1, No. 20. pp. 335-374.

BARRAT, James. 2013. La nostra invenzione finale. L’intelligenza artificiale a la fine dell'età dell' uomo. Nutrimenti SRL. Roma, Italia.

BAUMAN, Zygmund. 2005. Amor líquido. Acerca de la fragilidad de los vínculos humanos. Fondo de Cultura Económica. Buenos Aires, Argentina. 
BERMAN, Morris. 2006. Historia de la conciencia occidental: de la paradoja al complejo de autoridad sagrada. Editorial Cuatro Vientos. Santiago de Chile, Chile.

BETZU, Marco; COINU, Giovanni; DEMURO, Gianmario. 2019. "Gobernanza de los macrodatos y democracia representativa” En: Revista de Derecho Político. No. 106, pp. 253-264.

BOEHME-NEßBLER, Volker. 2016. "Privacy: a matter of democracy. Why democracy needs privacy and data protection" En: International Data Privacy Law. Vol. 6, No. 3, pp. 222-229.

BORBÓN RODRÍGUEZ, Diego Alejandro; BORBÓN RODRÍGUEZ, Luisa Fernanda; LAVERDE PINZÓN, Jeniffer. 2020. "Análisis crítico de los NeuroDerechos Humanos al libre albedrío y al acceso equitativo a tecnologías de mejora" En: Ius et Scientia. Vol. 6, No. 2, pp. 135-161.

CASTELLANOS CLARAMUNT, Jorge. 2019. "La democracia algorítmica: Inteligencia Artificial, Democracia y Participación Política” En: Revista General de Derecho Administrativo. No. 50, pp. 1-32.

CASTELLANOSCLARAMUNT, Jorge. 2020. “COVID-19, Intelligenza Artificiale e Democrazia: un’inevitabile difficoltà” En: Revista Multidisciplinar Humanidades e Tecnologias (FINOM). Vol. 26, pp. 38-55.

CORNEJO PLAZA, María Isabel. 2019. "El mejoramiento neural farmacológico neuroenhancement y el impacto en la esfera de los derechos humanos ¿un desafío para la democracia?” En: Valenzuela, Jonatan. (ed.) Desafíos Globales para la Democracia, ed. Tirant lo Blanche. Valencia, España, pp. 39-62.

DE TOCQUEVILLE, Alexis.1856/2015. Igualdad social y libertad política: antología esencial. Pierre Gibert. Madrid, España.

ESTAY SEPULVEDA, Juan Guillermo; LAGOMARSINO MONTOYA, Mario. 2020. Democracia al estilo búho Tercer Milenio: Miope y con cataratas. Editorial Cuadernos de Sofía. Ciudad de México, México.

FORNASIER, Mateus de Oliveira; BECK, Cesar. 2020. "Cambridge analytica: Escândalo, Legado e Possíveis Futuros para a Democracia” En: Direito em Debate. Ano XXIX, No. 53, pp. 182-195.

FOUCAULT, Michel. 2009. Vigilar y castigar. Nacimiento de la prisión. Editorial Siglo XXI, Ciudad de México, México.

GALLINA, Paolo. 2019. La mente luiquida. Come le macchine condizionano, modificano o potenziano il cervello. Edizione Dedado, Bari, Italia. 
Juan Guillermo Estay Sepúlveda, Mario Lagomarsino Montoya, Juan Mansilla Sepúlveda, Rosalba Mancina-Chavez, Alex Véliz Burgos y Alessandro Monteverde Sánchez

GÓMEZ PINEDA, Edisson. 2020. "Oportunidades, desafíos y nuevas perspectivas paradigmáticas de las administraciones públicas en tiempos de la cuarta revolución industrial" En: XXXIII Concurso del CLAD sobre Reforma del Estado y Modernización de la Administración Pública "La cuarta revolución industrial en la administración pública”. Caracas, Venezuela, pp. 1-21.

GONZÁLEZ DE LA GARZA, Luis Miguel 2018. "La crisis de la democracia representativa. Nuevas relaciones políticas entre democracia, populismo virtual, poderes privados y tecnocracia en la era de la propaganda electoral cognitiva virtual, el microtargeting y el Big Data” En: Revista de Derecho Político. No. 103, pp. 257-302.

GORWA, Robert; GUILBEAULT, Douglas. 2018. “Unpacking the Social Media Bot: A Typology to Guide Research and Policy" En: Policy and Internet. Vol. 12, No. 3, pp. 1-30.

HARDY, Robert Augustus; NORGAARD, Julia R. 2016. "Reputation in the Internet black market: an empirical and theoretical analysis of the Deep Web" En: Journal of Institutional Economics. Vol: 12, No. 3, pp. 515-539.

HEIDEGGER, Martin. 1950. Caminos de bosque. Alianza editorial. Madrid, España.

HERNÁNDEZ SELLÉS, Nuria. 2020. "Narrativas Transmedia: Fantasías animadas de ayer y hoy" En: Revista Indivisa. Boletín de Estudios e Investigación. No. 20, pp. 211-233.

JURNO, Amanda Chevtchouk; D’ANDRÉA, Carlos Frederico de Brito. 2017. "(In)Visibilidade algorítmica no "feed de notícias" do Facebook". En: Contemporánea Comunicação e cultura. Vol. 15, No. 2, pp. 463-484.

LEAL, Luziane de Figueiredo Simão. 2021. Inteligência Artificial nas Campanhas Eleitorais: a democracia das plataformas no banco dos réus. Editora Dialética. São Paulo, Brasil.

LIBEROS, Eduardo; NÚÑEZ, Álvaro; BAREÑO, Ruth; GARCÍA DEL POYO, Rafael; GUTIÉRREZ-ULECIA, Carlos; PINO, Gabriela. 2013. El libro de Marketing Interactivo y la Publicidad Digital. ESIC Editorial. Madrid, España.

LIMAOHIO.COM. 2021. Letter: What did Trump really say? 14 de enero de 2021. Disponible en línea. En: https://www.limaohio.com/opinion/ letters/443664/letter-what-did-trump-really-say. Fecha de consulta: $31 / 01 / 2021$. 
LIU, Xiao; MERRITT, Jeff. 2020. Shaping the Future of the Internet of Bodies: New challenges of technology governance. World Economic Forum. Geneva, Switzerland.

MADUREIRA, Gabriel Alarcon. 2019. "O Facebook enquanto "verdade seduzida": um ensaio crítico de sua comunidade global” En: Revista Extraprensa. Vol. 13, No. 1, pp. 129-144.

MATTELART, Armand; VITALIS, André. 2015. De Orwell al Cibercontrol. Editorial Gedisa. Barcelona, España.

MONASTERIO ASTOBIZA, Aníbal; AUSÍN, Txetxu; TOBOSO, Mario; MORTE FERRER, Ricardo; APARICIO PAYÁ, Manuel; LÓPEZ, Daniel. 2019. "Traducir el pensamiento en acción: Interfaces cerebro-máquina y problema ético de la agencia” En: Revista de Bioética y derecho. Perspectivas Bioéticas”. No. 46, pp. 29-46.

MORIN, Edgar. 1998. El pensamiento complejo. Editorial Gedisa. Barcelona, España.

MORSE, Stephen J. 2016. NeuroEthics: NeuroLaw. University of Pennsylvania Carey Law School. Disponible en línea. En https://scholarship.law. upenn.edu/faculty_scholarship/1726. Fecha de consulta: 28/01/2021.

MUÑOZ-MACHADO CAÑAS, Julia. 2020. "Noticias falsas. Confianza y configuración dela opinión pública en los tiempos deInternet” En: Corona Virus y otros problemas. El Cronista del Estado Social y Democrático de Derecho. No. 86-87. Portal Derecho S.A. Madrid, España, pp. 122-137.

MURTHY, Dhiraj; POWELL, Alison B.; TINATI, Ramine; ANSTEAD, Nick; CARR, Leslie; HALFORD, Susan J; WEAL, Mark. 2016. "Bots and Political Influence: A Sociotechnical Investigation of Social Network Capital” En: International Journal of Communication. No. 10 pp. 49524971.

MUSIC BUSINESS WORLD. 2021. Spotify's latest invention monitors your speech, determines your emotional state... and suggests music based on it. 27 de enero de 2021. Disponible en línea. En: https://inspiredsoundblog. wordpress.com/2021/02/01/spotifys-latest-invention-monitors-yourspeech-determines-your-emotional-state-and-suggests-music-basedon-it/. Fecha de consulta: 29/01/2021.

NEEF, Gina; NAGY, Peter. 2016. "Talking to Bots: Symbiotic Agency and the Case of Tay" En: International Journal of Communication. Vol. 10, pp. 4915-4931.

NORTE SOSA, José. 2020. BIG DATA Comunicación y Política: De la democracia a la cybercracia. Editorial Kier. Buenos Aires, Argentina. 
Juan Guillermo Estay Sepúlveda, Mario Lagomarsino Montoya, Juan Mansilla Sepúlveda, Rosalba Mancina-Chavez, Alex Véliz Burgos y Alessandro Monteverde Sánchez

ORSOLINI, Laura; PAPANTI, Duccio; CORKERY, John; SCHIFANO, Fabrizio. 2017. "An insight into the deep web; why it matters for addiction psychiatry? En: Human Psychopharmacology: Clinical and Experimental. Vol. 32, No. 3. Disponible en línea. En: https://doi. org/10.1002/hup.2573. Fecha de consulta: 25/08/2020.

PARRA VALERO, Pablo; OLIVEIRA, Lídia. 2018. "Fake news: una revisión sistemática de la literatura" En: Observaorio OBS. Special Issue, pp. 5478.

PERUCCIETTI, Enrica. 2019. Cyberuomo. Dall'intelligenza artificiale all'ibrido uomo-macchina. Arianna Editrice. Bologna, Italia.

PETIT, Martí. 2018. "Por una crítica de la razón algorítmica. Estado de la cuestión sobre la inteligencia artificial, su influencia en la política y su regulación” En: Quaderns del CAC. Vol, XXI, No. 44, pp. 5-15.

REIG, Ramón. 2020. Evolución, historia y comunicación en un mundo digital (discurso hipotético). Editorial Anthopos. Barcelona, España.

RODRÍGUEZ FERRÁNDEZ, Samuel. 2018. "Investigación neurocientífica y derecho penal". En: Teoría y derecho: Revista de pensamiento jurídico. No. 23, pp. 186-208.

ROSENBACH, Eric; Mansted, Katherine. 2018. Can Democracy Suvervi in the Information Age? Harvard Kennedy School. Belfer Center for Science and International Affairs. Massachussets, Estados Unidos.

SALAS ABAD, Carlos. 2019. "La primera fake news de la historia" En: Historia y comunicación social. Vol. 24, No. 2, pp. 411-431.

SÁNCHEZ BARBA, Gabriela A. 2014. We are Biohackers. Exploring the collective identity of the diybio movement. Master of Science Thesis. Delft University of Technology. Geneve, Italia.

SÁNCHEZ MEDERO, Gema. 2015. "El ciberterrorismo: de la web 2.o al Internet profundo” En: Revista Ábaco. Vol. 3, No. 85, pp. 100-108.

TRUJILLO, Cleber A; RICE, Edwards, S; SCHAEFER, Nathan K; CHAIM, Isaac A; WHEELER, Emily C; MADRIGAL, Assael A; BUCHANAL, Justin; PREISSL, Sebastián; WANG, Allen; NEGRAES, Priscilla D; SZETO, Ryan A; HERAI, Roberto H; HUSEYNOV, Alik; FERRAZ, Mariana SA; BORGES, Fernando S; KIHARA, Alexandre H; BYRNE, Ashley; MARIN, Maximilian; VOLLMERS, Christopher; BROOKS, Angela; LAUTZ, Jonathan D; SEMENDEFERRI, Katerina; SHAPIRO, Beth; YEO, Gene W; SMITH, Stephen E; MOUTRI, Alysson R. 2021. "Reintroduction of the archaic variant of NOVA1 in cortical organoids alters neurodevelopment" 
En: Science. Disponible en línea. En: DOI: https://doi.org/10.1126/ science.aax2537. Fecha de consulta: 08/09/2020.

VALE, Do Simone. 2016. "Inteligência Artificial \& Redes Sociais: notas sobre um bot que odiava humanos" En: Áquila. No. 15, pp. 36-48.

VALERA-ORDAZ. Lidia. 2019. "Liberal Individualist, Communitarian, or Deliberative? En: International Journal of Communication. Disponible en línea. En: https://ijoc.org/index.php/ijoc/article/view/8309/2584. Fecha de consulta: 14/06/2020.

VELOSO, Maria do Socorro Furtado Veloso; LOPES, John Willian. 2020. "Excursões ao ciberativismo: uma análise de compreensões conceituais e tipológicas" En: Contemporánea. Comunicação e cultura. Vol. 18, No. 1, pp. 157-1 

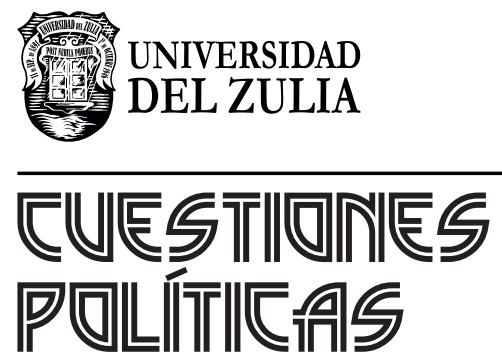

Vol.39 No 68

Esta revista fue editada en formato digital y publicada en enero de 2021, por el Fondo Editorial Serbiluz, Universidad del Zulia. Maracaibo-Venezuela 